\title{
Le calendrier du Yatenga
}

The calendar of Yatenga

Michel Izard

\section{OpenEdition}

Journals

Édition électronique

URL : http://journals.openedition.org/span/559

DOI : 10.4000/span.559

ISSN : 2268-1558

\section{Éditeur}

École pratique des hautes études. Sciences humaines

\section{Édition imprimée}

Date de publication : 1 septembre 1986

Pagination : 45-55

ISSN : 0294-7080

\section{Référence électronique}

Michel Izard, « Le calendrier du Yatenga », Systèmes de pensée en Afrique noire [En ligne] 7 | 1986, mis en ligne le 05 juin 2013, consulté le 19 avril 2019. URL : http://journals.openedition.org/span/559 ; DOI : 10.4000/span.559 


\title{
LE CALENDRIER DU YATENGA
}

\section{par}

\author{
Michel Izard
}

\begin{abstract}
"Le soleir, la Lumière se lève à l'orient. Mais la Zumière n'est que le simple rapport à elze-même; la lumière en soi universelze est aussi sujet, dans le soleil. On a souvent décrit la scène d'un aveugle qui, recouvrant soudain la vue, apercoit l'aube, la Zumière qui vient et le soleil qui flamboie. D'abord, c'est à la vue de cette clarté, l'oubli infini de soi-même, l'admiration absolue. Toutefois, à mesure que le soleiz s'élève, cette admiration s'atténue; on apergoit les objets environnants, et de ceux-ci l'on descend dans son propre for intérieur; et ainsi le progrès devient un rapport réciproque. Alors l'homme passe d'une contemplation inactive à l'activité et au soir, ir a bâti un édifice formé avec un soleir intérieur; et quand au soir, il le considère, il en fait plus de cas que le premier soleit, extérieur. Car maintenant, il se trouve en relation ave son esprit et par la suite dans une condition libre. Tenons ferme cette image, elle contient déjà le cours de l'histoire universelze, la grande journée de l'esprit".

Hege1, Lecons sur la phizosophie de l'histoire, trad. Gibelin
\end{abstract}

Le calendrier du Yatenga (1) associe une année solaire (yumde) et des mois lunaires ( $k i i d u$, sing. kiugu, terme qui désigne la lune). Le mois lunaire est délimité par deux retours consécutifs de 1a lune en conjonction avec le soleil; il correspond à une révolution synodique de la lune, de durée moyenne 29 jours 12 heures 44 minutes, avec un écart maximum à la moyenne de $+/-6$ heures 30 minutes, lié à 1 'interférence des excentricités propres aux orbites solaire et lunaire. Ce ne sont pas les intervalles entre deux nouvelles lunes qui sont dénommés, mais les nouvelles lunes elles-mêmes, de sorte que le comput du temps est un décomptè de jours par rapport à la nouvelle lune : jours précédant, jours 
suivant la nouvelle lune. Astronomiquement parlant, la nouvelle lune (kitika) correspond au passage de la lune au même méridien que le soleil, la lune et le soleil ayant alors même ascension droite. Il suit que 1a nouvelle lune astronomique, à s'eñ tenir à la définition même du phénomène, est invisible à l'oeil nu, sauf en cas d'éclipse, totale ou partielle. Le kilika correspond donc à la nouvelle lune visible. Compte tenu que la lune se déplace par rapport aux étoiles d'environ $13^{\circ}$ par 24 heures et qu'elle n'est visible, dans de bonnes conditions atmosphériques, que si elle est à environ $15^{\circ}$ du soleil couchant, la nouvelle. Iune visible suit d'au moins 24 heures la nouvelle lune astronomique. Une année solaire voit se répéter douze fois le phénomène de la nouvelle lune; il y a ainsi douze lunaisons par année solaire, avec un retard de l'année lunaire sur 1 'année solaire (épacte) de 10,87 jours : il y a donc coincidence approximative entre la durée de trois années solaires et celle de trois années lunaires augmentée d'une lunaison, soit trente-sept mois lunaires. Pour maintenir les mois lunaires dans une position approximativement stable sur l'année solaire, par quoi se manifeste précisément le caractère à la fois solaire et lunaire du calendrier, il est nécessaire et suffisant d'intercaler un mois lunaire supplémentaire après trois années solaires révolues : on a alors des cycles de trois années solaires tels que les deux premières comptent douze lunaisons et la troisième, treize. Dans le calendrier du Yatenga, le mois Iunaire intercalaire (embolisme) est introduit toutes les trois annēes solaires par doublement de la sixième lunaison, appelée kizalogo ou "Iune vide". Il est remarquable que 1 'introduction du mois lunaire supplémentaire intervienne en début de saison des cultures, vers les mois de mai et juin de notre calendrier; cette opération a pour effet immédiat de réduire 1 'avance généralement prise par les travaux agricoles sur les lunaisons de la saison des pluies dont les noms rappellent les principales étapes des activités culturales (2).

L'année solaire commence avec l'apparition de la nouvelle lune 1a plus proche du moment de plus grande déclinaison australe du soleil (solstice d'hiver). Les noms des douze nouvelles lunes sont les suivantes : 1. filiga; 2. gambo; 3. womson; 4. bugure; 5. gursi; 6. Kunduba; 7. kizalogo; 8. budubo; 9. warga; 10. banka; 11. yaalum; 12. tiido; rappelons que tous les trois ans le mois lunaire de kizalogo a une durée de deux lunaisons. 
Le terme fiziga (employé dans le même sens au pluriel : filise) signifie "remerciement"; au Nouvel An, plutôt que de former des voeux pour le déroulement de l'année qui commence, on remercie la terre et les ancêtres pour les bienfaits prodigués par 1'année qui vient de s'achever. A l'apparition "officielle" de la lune de filiga, on célèbre dans tout le royaume la fête du même nom, aussi appelée fitikeenga, "grand filiga", qui est suivie par les trois salutations annuelles au roi (napusum) des trois ordres de détenteurs du pouvoir (naam), chaque ordre ayant son propre napusum : les nayirdemba (serviteurs royaux), les tãsobnamba (maîtres de la guerre) et les nakombse (membres de 1'aristocratie royale); entre les deux premiers napusum (nayirciemba, tãsobnamba) se déroulent les cérémonies royales nocturnes du siimde, destinées à honorer la mémoire des ancêtres royaux. Les cinq lunes suivantes portent les noms de cinq localités du royaume : Gambo, Womson, Bugure, Gursi, Kunduba. C'est au long de la période couverte par ces cinq lunaisons qu'ont lieu dans les villages les fêtes de fertilité qui constituent ensemble le cycle du bega. C'est le roi qui, à l'approche de chaque nouvelle lune de la période du bega, ouvre la phase correspondante du cycle cérémoniel en faisant remettre au mâttre de la terre (tengsoba) de la localité éponyme du mois lunaire du sel et des graines de néré (Parkia biglobosa, roãga), qui sont partagés par le destinataire de ces dons entre tous les maîtres de la terre de son obédience. Le roi donne les "condiments" de la sauce, aux gens de la terre (tengdemba) d'obtenir le mil; après les récoltes, le mil nouveau, sous forme de bouquets d'épis de sorgho, sera offert au roi à l'occasion des napusum, apporté par les chefs des villages. Après les lunes du bega vient la période de kizalogo qui a une durée d'une ou deux lunaisons; Kizalogo est situé à 1 'articulation des lunes cérémonielles de saison sèche (11e et $12 \mathrm{e}$, lère à $6 \mathrm{e}$ ) et des lunes de la période des travaux agricoles ( 8 e à 11e), la onzième lune, comme on peut le constater, correspondant à la seconde des deux articulations entre les deux grandes divisions de l'année, à ceci près que quand kizalogo n'appartient fondamentalement à aucune de ces deux périodes, ycalum appartient pratiquement aux deux. Les trois lunes suivantes portent les noms des trois principales phases de la culture du mil : budubo, les senailles; warga, le premier sarclage; banka, le second sarclage ou binage. En réalité, ces opérations culturales n'ont généralement pas lieu dans les 
mois qui en portent les noms, mais sensiblement avant. Nous avons montré ailleurs (3) que sur la période de dix années allant de 1953 à 1962 , la chute de la première pluje de 1 'hivernage est intervenue entre un 22 mars et un $15 \mathrm{mai}$, or, si les premières pluies sont abondantes, on sème immédiatement, de telle manière qu'au plus tard, sauf en cas de grave retard des premières précipitations de la saison humide, les semailles ont lieu vers la fin de mai ou le début de juin. Si l'on sème en juin, on sarcle en juillet et $I^{\prime}$ on bine en aôtt; ainsi les semailles ont plutôt lieu en kizalogo, le sarclage en budubo et le binage en warga, le mois de banka étant celui du mûrissement du mil, pendant lequel les travaux agricoles sont au point mort pour l'essentiel, puisque la seule tâche alors à accomplir est la récolte du maîs hâtif cultivé à la périphérie immédiate des habitations. Le terme par lequel est appelée la onzième lune -- yaalum -- désigne le mil (en l'occurence le sorgho blanc, kenda) qui a atteint la taille de la maturité sans qu'il y ait eu formation de grain, et donc d'épis, du fait de pluies tardives trop abondantes dont n'a résulté qu'une croissance sans mûrissement; en cette période d'attente, trop souvent anxieuse, où la qualité de la récolte dépend d'un petit nombre de chutes d'eau normalement abondantes et bien réparties sous un ensoleillement déjà vif, la hantise d'une mauvaise récolte est ainsi conjurée. C'est après l'apparition de la lune de yaalum qu'a lieu la fête royale de koom filiga, "filiga de l'eau" (mais koom, "eau", désigne ici la bière de mil, raam), qui associe le roi et les mâtres de la guerre; la célébration du koom filiga permet au roi de consommer le mil de la nouvelle récolte. L'année s'achève avec la lune de tiido, d'un mot qui, sous la forme du pluriel (1e singulier tiibo est peu usité dans le Yatenga) désigne à la fois le second des trois grands regalia du Yatenga (naam tiido) et, c'est le cas ici, 1 'autel des buguba, prêtres maîtres de la pluie et gardiens de l'esprit du mil. A l'apparition de la lune de tiido, les buguba célèbrent une fête qui porte le même nom, à l'occasion de laquelle est levé l'interdit de la consommation de 1 'eau des puits, qui pesait sur eux depuis la première chute de pluie de I'hivernage.

Soit I'année 1964. Comparons les moments d'apparition des nouvelles Iunes astronomiques et des nouvelles lunes visibles : 


\begin{tabular}{llrl} 
mois & \multicolumn{2}{l}{ lune astronomique } & lune visible \\
gambo & 14 janvier & $20 \mathrm{~h} 44$ & 15 janvier \\
womson & 13 février & $13 \mathrm{~h} 02$ & 15 février \\
bugure & 14 mars & $2 \mathrm{~h} 14$ & 16 mars \\
gursi & 12 avril & $12 \mathrm{~h} 38$ & 14 avril \\
kunduba & 11 mai & $21 \mathrm{~h} 02$ & 12 mai \\
kizalogo * & 10 juin & $4 \mathrm{~h} 23$ & 12 juin \\
budubo & 9 juillet & $11 \mathrm{~h} 31$ & 11 juillet \\
warga & 7 août & $19 \mathrm{~h} 17$ & 8 août \\
banka & 6 septembre $4 \mathrm{~h} 35$ & 8 septembre \\
yaalum & 5 octobre & $16 \mathrm{~h} 20$ & 7 octobre \\
tiido & 4 novembre & $7 \mathrm{~h} 17$ & 6 novembre \\
filiga & 4 décembre & $1 \mathrm{~h} 19$ & 6 décembre \\
* Durée d'une lunaison & &
\end{tabular}

Notons que, dans la colonne de droite, nous n'avons que des dates indicatives de visibilité possible de la lune; d'un point à l'autre du royaume, on pourra trouver des différences d'une journée, exceptionnellement de deux, entre les dates d"apparition" de la lune, que cette apparition soit localement retardée du fait de conditions atmosphériques défavorables ou qu'une raison rituelle en fasse différer l'annonce. Le moment officiel -- pour tout le royaume -- de l'apparition de la lune du Nouvel An ( $f i l i g a$ ) est annoncé par un coup de fusil tiré depuis le sanctuaire royal du Yikeemde, à Ouahigouya, résidence du roi, auquel répond immédiatement un autre coup de fusil, tiré depuis un endroit situé en brousse et appelé Kiugẽ, le "lieu de la lune", à l'est de Ouahigouya, où le roi fait retraite; 1'annonce de l'apparition de la lune est en principe aussitôt transmise de village en village, mais comme on 's'efforce de faire intervenir cette apparition dans la nuit d'un samedi à un dimanche (le Yatenga utilise la semaine arabe de sept jours) ou dans une autre nuit précédant un jour faste, tout le monde sait d'avance quand la lune doit "sortir". Les apparitions des lunes du bega, de yaalum (fête de koom fiziga) et de tiido font également 1 'objet d'une annonce officielle, car elles sont marquées par des manifestations qui associent respectivement les mâ̂tres de la terre, les maîtres de la guerre, les buguba, et le roi. 
Si nous pointons sur la carte les villages de Gambo, Womson, Bugure, Gursi et Kunduba, nous pouvons tracer un périmètre fermé reliant de proche en proche ces cinq villages selon l'ordre du calendrier et tel qu'il donne, si on le décrit de Gambo à Gambo, le parcours circulaire orienté que les Moose du Yatenga appellent napusumgizgri, circumambulation ( $g i$ Igri) s'effectuant dans le sens des aiguilles d'une montre que l'on trouve intégré dans les rituels royaux sous la forme d'un déplacement semi-circulaire ou circulaire autour du palais royal, de la porte de 1 'est (porte des femmes et des captifs) à la porte de l'ouest (porte des Moose) par le nord, ou de la porte de l'est à la porte de l'est par le nord et le sud, donc par la porte de 1 'ouest, et qui correspond aussi au sens dans lequel le roi se déplace de village en village au cours de son voyage d'intronisation, le ringu (4).

Le nopusumgizgri, sens "normal" de circumambulation rituelle, dont le nom évoque les fêtes de salutation au roi (napusum) qui suivent 1e Nouvel An, s'inverse en yirgizgri, circumambulation de la "maison" (yiri) qui intervient notamment dans les rituels funéraires royaux : ainsi, avant de transporter le cadavre du roi du palais royal (nayimi) au cimetière royal (nayaado), lui fait-on faire trois tours complets de l'enceinte de la résidence royale depuis la porte de l'ouest et de telle manière que 1 'on atteigne la porte de l'est par le nord (napusumgizgri : d'ouest en est par le sud). Ces parcours circulaires imposent l'image d'un royaume assimilable à un cercle dont le centre est le lieu ò̀ se tient le roi. Plus précisément, nous ne disons pas que les Moose du Yatenga envisagent, ou se représentent, le territoire de leur royaume comme un espace enclos par un cercle, mais que le rapport symbolique à l'espace du royaume s'établit par le moyen rituel d'une inscription circulaire sur le sol. Ainsi, le roi prend possession de son royaume au cours d'un voyage dont le nom, ringu, signifie "royaume", et son itinéraire décrit un cercle : c'est ici la périphérie qui détermine le centre.

La circularité du napusumgizgri et du yirgilgri n'est pas dissociable de l'existence d'un axe de l'espace-sol orienté d'est en ouest, le sens est-ouest étant celui de la vie, le sens inverse ouest-est celui de la mort. Pour employer le langage de la géométrie, on pourrait dire qu'il existe une relation de transformation réciproque entre 1'axe estouest et tout cercle orienté dans le sens des aiguilles d'une montre 
dont le centre est un point de la droite supportant l'axe, et que cette relation s'inverse en une transformation de même nature mais de sens opposé, associant l'axe ouest-est à tout cercle orienté dans le sens inverse des aiguilles d'une montre, la condition concernant la position du centre étant inchangée, les deux axes ayant même support. Dans un plan perpendiculaire à celui du sol, et dont la projection sur le sol est l'axe est-ouest, il est un autre déplacement circulaire, ou plutôt semi-circulaire, remarquable : celui du soleil dans le ciel, d'est en ouest. De son lever à son coucher, en passant par son zénith, le soleil décrit une trajectoire dont on peut considérer qu'elle fait l'objet, sur le sol, d'une double "projection" : 1'axe est-ouest, d'une part, le demi-cercle est-nord-ouest, d'autre part, cette seconde projection consistant en un rabattement du demi-cércle de la trajectoire solaire sur le plan horizontal (sol), au moyen d'une rotation de $90^{\circ}$ autour de 1 'axe est-ouest, de sens zénith-nord. On peut conclure de ce qui précède que le monde mooga est sphérique. Nous avons une sphère partagée deux fois en deux : par le plan vertical défini par la trajectoire du soleil et par le plan du sol, l'axe est-ouest étant l'intersection de ces deux plans. Le centre de la sphère définit la place du roi, centre du monde dont le lieu sur le sol est déterminé par la projection géométrique de l'axe vertical du monde, qui associe donc le roi sur la terre et le soleil à son zénith dans le ciel. La sphère du monde, dont nous avons fixé le centre et les axes remarquables, est définie, pour ce qui est de ses limites (de son rayon) par deux cercles également remarquables : celui qui détermine le plan du sol et sur lequel le sens normal de parcours définit l'espace du royaume, et le cercle auquel appartient le demi-cercle de la trajectoire visible du soleil, que complète le demicercle d'une trajectoire invisible de même sens, par laquelle le soleil opère un retour régulier au point de départ de sa course diurne, situé à l'orient. Sur cette sphère, le cercle que nous avons précédemment introduit comme support du quadrant de rabattement du zënith sur le nord, et dont le plan est perpendiculaire à la fois au plan du sol et à celui de la trajectoire solaire, n'a pas de statut cosmologique, tout comme 1 'axe nord-sud, sur le.sol, $n$ 'a pas de statut ontologique. Dans cet univers sphérique, on peut dire que le "centre est partout et la circonférence nulle part", en ce que, d'une part, il n'y a centre que pour autant qu'il y a coincidence entre un être (le roi) et une place, et que 
d'autre part, il y a homologie entre des sphères de même centre mais à rayon variable et les divers cercles qu'actualise la vie du royaume. Le roi n'occupe le centre du monde que pour autant que sa présence à ce monde y exemplifie toute présence humaine : c'est en ce sens que le centre est partout, à la fois lieu et moment, car c'est dans la crainte et le tremblement du milieu du jour, à l'heure où les êtres et les choses apparaîssent dans leur nue vérité de n'avoir pas d'ombre, que I'homme éprouve sa condition singulière, entre vie et mort, entre passé et futur, quand, ici et maintenant, il se tient debout, face à 1 'ouest. De 1 'homme ordinaire, faire retour au roi, c'est situer 1 'itinéraire -1 'axe du monde -- qui conduit du "pouvoir" divin (wennaam) au pouvoir humain (naam), généalogie qui justifie, à 1'inverse, que Wende, le dieu qui est au ciel, soit salué du titre de "chef" (naaba); c'est aussi poser l'homologie entre la situation du soleil dans le ciel, qui conduit à identifier l'astre diurne et Naaba Wende, et celle du roi sur la terre; c'est enfin, sur l'espace-sol, définir l'opérateur général d'articulation entre l'univers solaire du pouvoir et l'univers lunaire de la terre.

Mais revenons au mois du bega. A chacun des cinq villages éponymes correspond une maîtrise de la terre supérieure, de telle sorte que ces cinq maîtrises de la terre se partagent 1 'espace entier du royaume comme autant de secteurs se partageraient un espace circulaire. Compte tenu que c'est le roi qui donne l'impulsion initiale à chacune des cinq phases de ce grand rituel de fertilité qu'est 1 e bega, nous retrouvons bien le souverain à l'articulation des mondes du pouvoir et de la terre. A nouveau, le roi est au centre d'un dispositif circulaire, mais qui se distingue cependant de ceux que nous avons mis jusqu'ici en évidence par son mode de "construction". Le fait que le roi ait ici pour partenaires "périphériques" cinq hauts dignitaires de la terre exclut toute référence à un axe de l'espace-sol; par contre, cette configuration étoilée surdétermine l'existence d'un axe du monde, perpendiculaire au plan de l'espace-sol en un lieu qui est celui où se tient le roi. Ce qui est nouveau, avec le bega, c'est qu'il n'y a plus solution de continuité entre le centre et la périphérie, mais au contraire possibilité de passage de proche en proche du centre vers la périphérie, par "remplissage" de l'espace au moyen d'une multiplication de parcours, ceux suivis, de village en village, par les manifestations locales. 
La résultante de ces mouvements est de sens centre-périphérie, qui s'inverse au moment des salutations annuelles au roi, quand le mil obtenu grâce à 1 'accomplissement des rituels de fertilité est transporté des villages à la résidence royale.

Le roi est sur l'espace-sol comme le soleil zénithal est dans le ciel diurne; il est le "centre" d'un cercle que l'on peut définir comme la projection du ciel diurne sur la terre, mais ce centre n'engendre pas la circonférence qui lui est associée, il n'est que le lieu punctiforme par lequel passe l'axe est-ouest de l'espace-sol. La lune n'occupe pas dans le ciel nocturne une position comparable à celle du soleil dans le ciel diurne; elle n'intervient pas dans la détermination du centre de l'espace-sol circulaire, non plus que dans celle de son axe. Par contre, c'est la projection du mouvement de la lune sur l'espace-sol qui en définit le pourtour circulaire : on a vu que ce cercle est décrit, dans le cours d'une année solaire, au long de cinq mois consécutifs, les villages éponymes des nouvelles lunes correspondantes décrivant, sur le territoire du royaume, un cercle dont le centre est le roi. Le cycle du bega nous montre que la lune est liée à la sacralité de la terre, tandis qu'au soleil est associée l'idéologie du pouvoir. Le pouvoir et la terre ont respectivement leur spatialité et leur temporalité propre. La spatialité du pouvoir est construite à partir de ponctuations et de linéarisations, tandis que celle de la terre procède d'un donné d'espace plein, c'est une spatialité de surfaces. La temporalité du pouvoir est celle de 1 'histoire, y compris de 1 'histoire individuelle; le temps du pouvoir est borné et cumulatif, tandis que le temps de la terre, cyclique par excellence, est répétitif et sans fin. Le calendrier du Yatenga rend compte de l'articulation nécessaire entre deux temporalités, qui est articulation aussi entre deux spatialités; le temps n'est pas pensable, mesurable, hors de l'espace.

Michel Izard

Directeur de recherche au C.N.R.S. Laboratoire d'anthropologie sociale (Collège de France) 
NOTES

(1) Le Yatenga est 1 'un des principaux royaumes moose (sing. mooga) du bassin de la Volta Blanche, constituant le Moogo et dominés par les Moose. Nous avons précédemment traité du calendrier du Yatenga dans: Les archives orales d'un royaume africain. Recherches sur la formation du Yatenga, Paris, 1980, 2 t., multigr., t. I : 349-356. On comparera le calendrier mooga du Yatenga avec le très proche calendrier kurumdo (fulga) du Lurum : cf. Annemarie Schwegel-Hefel et Wilhelm Staude, Die Kummba von Lurum. Monographie eines Volkes aus Obervolta (Westafrika), Wien, 1972, 98-106.

(2) I1 est possible que la régulation de l'année lunaire soit associée à 1'observation des Pléiades, mais nous ne disposons pas d'informations surce point. Jean-Marie Kohler (Activités agricoles et changements sociaux dans l'Ouest Mossi, Paris, 1971 : 46) écrit à ce propos : "Quelques auteurs rapportent que les cultivateurs mossi se fient aux constellations pour déterminer le moment favorable aux semailles. L'enquête dans 1 'Ouest a révélé que les cultivateurs de cette région ne s'entendent pas à ce sujet, ce qui révèle sans doute le peu d'importance pratique qu'ils accordent aux mouvements des astres pour la conduite de leur activités agricoles". Nous pourrions faire la même remarque pour ce qui concerne le Yatenga, ce qui n'épuise cependant pas le sujet. Lambert (Le pays mossi et sa population. Etude historique, économique et géographique suivie d'un essai d'ethnographie comparée, 1907, inédit, ms. : 218-219) écrit : "Pour les Mossi (...) les époques où il convient d'entreprendre les travaux des champs sont réglées par le retour périodique des étoiles. Au mois de mai, lorsque Cassiopée (en mossi : boudoubakoukouri, le manche à semer) se montre au N.E. avant le lever du soleil, le défrichage doit être terminé (...). Pour le kaserba (gros mil rouge, M.I.), les semailles doivent être terminées lorsque les Pléiades (nommées sousi, les crins de cheval) se montrent à l'est. Les autres graines peuvent encore être mises en terre avec la certitude d'obtenir un résultat; pour déterminer cette période de répit d'une façon précise, les Mossi usent d'un procédé curieux. A 1'aube, lorsque les étoiles sont sur le point d'être complètement éclipsées par la lumière du soleil levant, il faut pouvoir regarder les pléiades avec une pierre sur la tête; si cet astérisme est encore assez bas sur 1 'horizon pour que 1 'observateur 1 'aperçoive sans que la pierre retombe, il sera possible de semer encore avec fruit. Le sésame (sini) ne doit être semé qu'après l'apparition des Pléiades". En note, Lambert ajoute : "Les Mossi possèdent encore d'autres connaissances cosmographiques. Ils nomment la Voie Lactée ioumtaodora (limite de l'année), parce que, disent-ils, au moment où finit l'année, elle partage exactement le ciel en deux. Ils considèrent les cinq étoiles de la Grande Ourse comme une constellation qu'ils appellent tatoba (les tireurs). Ils ne distinguent pas les étoiles des planètes et, de celles-ci, ils ne connaissent que Vénus dans laquelle ils voient, selon l'époque de l'année, deux étoiles différentes : ibeorho-wadrha l'étoile du matin, et saralhisere-wardha, l'étoile du repas du soir. Les comètes, wad-zoure (étoile à queue), les impressionnent beaucoup (...)3 Citons encore Robert Pageard ("Recherches sur les Nioniossé", Etudes voltaiqques, nouvelle série, 4, 1963, 5-71: 53) : "Le "boudoub-koukouri" serait une représentation de Yabr'Pandé, I'ancêtre 
féminin dont le nom signifie "fil de trame". "Boudoub'koukouri" est également le nom donné à la constellation des Pléiades dont la position dans le ciel règle les semences dans toute la savane ouest-africaine. La relation Pandé (femme) - terre nourricière est normale. Nous pensons que les Pléiades pourraient elles-mêmes être en rapport avec la matrice et les graines : cela oblige à penser à la calebasse qui contient les graines à semer, "boud-gyebgha".

(3) Op. cit., 1980, t. I : 27-28.

(4) L'habitation mooga est délimitée par un mur d'enceinte sur lequel s'adossent, à l'intérieur, des chambres circulaires; le mur comprend une ouverture, à 1 'ouest. L'habitation du roi (nayiri), de très grande taille et dont l'organisation intérieure est extrêmement complexe, comprend deux ouvertures : la porte large de l'est, réservée aux épouses royales et aux captifs royaux (dignitaires et serviteurs), et la porte royale de 1 'ouest, réservée au roi et aux Moose (dignitaires et serviteurs). Sur toutes les questions seulement évoquées dans ce court article, on peut se reporter à notre ouvrage Gens du pouvoir, gens de $2 \alpha$ terre. Les institutions politiques de l'ancien royaume du Yatenga (Bassin de La Volta Blanche), Cambridge-Paris, 1985, notamment chap. 2 et $3: 71-204$. 